\section{(2) OPEN ACCESS}

\title{
Impact of COVID-19 national lockdown on asthma exacerbations: interrupted time-series analysis of English primary care data
}

\author{
Syed A Shah, ${ }^{1}$ Jennifer K Quint 지, ${ }^{2}$ Bright I Nwaru 지 , ${ }^{1,3}$ Aziz Sheikh ${ }^{1}$
}

\begin{abstract}
- Additional material is published online only. To view please visit the journal online (http://dx.doi.org/10.1136/ thoraxjnl-2020-216512).
\end{abstract}

'Asthma UK Centre for Applied Research, Centre for Medical Informatics, Usher Institute, The University of Edinburgh, Edinburgh, UK

${ }^{2}$ National Heart \& Lung Institute, Imperial College London, London, UK ${ }^{3}$ Krefting Research Centre, The University of Edinburgh, Gothenburg, Sweden

Correspondence to Dr Syed A Shah, Asthma UK Centre for Applied Research, Centre for Medical Informatics, Usher Institute, The University of Edinburgh, Edinburgh, UK; Ahmar.Shah@ed.ac.uk

Received 2 November 2020 Revised 7 December 2020 Accepted 11 January 2021 Published Online First 29 March 2021

\section{Linked}

- http://dx.doi.org/10.1136/ thoraxjnl-2020-216380 - http://dx.doi.org/10.1136/ thoraxjnl-2020-216526 - http://dx.doi.org/10.1136/ thoraxjnl-2021-216930

Check for updates

(C) Author(s) (or their employer(s)) 2021. Re-use permitted under CC BY. Published by BMJ.

To cite: Shah SA, Quint JK Nwaru $\mathrm{Bl}$, et al. Thorax 2021:76:860-866.

\section{ABSTRACT}

Background The impact of COVID-19 and ensuing national lockdown on asthma exacerbations is unclear. Methods We conducted an interrupted time-series (lockdown on 23 March 2020 as point of interruption) analysis in asthma cohort identified using a validated algorithm from a national-level primary care database, the Optimum Patient Care Database. We derived asthma exacerbation rates for every week and compared exacerbation rates in the period: January to August 2020 with a pre-COVID-19 period and January to August 2016-2019. Exacerbations were defined as asthma-related hospital attendance/admission (including accident and emergency visit), or an acute course of oral corticosteroids with evidence of respiratory review, as recorded in primary care. We used a generalised least squares modelling approach and stratified the analyses by age, sex, English region and healthcare setting.

Results From a database of 9949387 patients, there were 100165 patients with asthma who experienced at least one exacerbation during 2016-2020. Of 278 996 exacerbation episodes, 49938 (17.9\%) required hospital visit. Comparing pre-lockdown to post-lockdown period, we observed a statistically significant reduction in the level $(-0.196$ episodes per person-year; $p<0.001$; almost 20 episodes for every 100 patients with asthma per year) of exacerbation rates across all patients. The reductions in level in stratified analyses were: 0.005 0.244 (healthcare setting, only those without hospital attendance/admission were significant), 0.210-0.277 (sex), 0.159-0.367 (age), 0.068-0.590 (region).

Conclusions There has been a significant reduction in attendance to primary care for asthma exacerbations during the pandemic. This reduction was observed in all age groups, both sexes and across most regions in England.

\section{INTRODUCTION}

Early studies from Wuhan City (China), where COVID-19 caused by SARS-CoV-2 was first identified in December 2019, suggested that COVID-19 can lead to severe pneumonia and life-threatening acute respiratory distress syndrome. ${ }^{1}$ These initial investigations also demonstrated that people with underlying health conditions, including respiratory illnesses, are at higher risk of severe disease and death due to COVID-19. ${ }^{2}$ These findings were further corroborated by studies in the USA, ${ }^{34} \mathrm{UK}^{56}$ and other European countries.

\section{Key messages}

What is the key question?

$\Rightarrow$ What is the impact of COVID-19 and the ensuing national lockdown on healthcare attendance for asthma exacerbations?

What is the bottom line?

$\Rightarrow$ Our national-level, interrupted-time series study following a cohort of 100165 patients with asthma across England showed that there was a substantial reduction in overall primary care-recorded exacerbation rates for both men and women, in all age groups and across most regions in England. This reduction was observed in exacerbations managed in primary care that did not require a hospital visit. Our study found no significant change in more serious exacerbations that required hospital attendances and/or admission.

Why read on?

$\Rightarrow$ Existing evidence suggests that having a comorbidity such as asthma is a risk factor of COVID-19-related hospitalisation and possibly death. However, the impact of the pandemic and the ensuing national lockdown on asthma exacerbations is not clear. This study provides national-level insights about the impact of national lockdown measures on healthcare attendance for asthma exacerbations.

Asthma has been identified as a possible risk factor for COVID-19-associated hospitalisation and death. ${ }^{36}$ A significant proportion of asthma-related healthcare resource utilisation and cost is associated with asthma exacerbations. ${ }^{8}$ Overall, asthma exacerbations represent a huge socioeconomic burden both in the UK and across the world. In the UK alone, there are at least 6.3 million primary care consultations, 93000 hospital episodes and 1400 deaths attributed to asthma every year. ${ }^{9}$

Since the majority of asthma exacerbations are associated with respiratory viral illnesses, ${ }^{10}$ there was initial concern that COVID-19 may lead to increased asthma exacerbations. ${ }^{11}$ However, the evidence to date is not clear. A study from Wuhan reported that only 0.9\% patient among the 269 severe cases investigated had asthma. ${ }^{12}$ Another study from Paris (France) reported that patients with asthma were not over-represented among the 768 hospitalised patients with COVID-19. ${ }^{13}$ 
However, some studies have suggested that the percentage of patients who have asthma among patients with COVID-19 who are hospitalised is higher than asthma prevalence. ${ }^{14} 15$ In the largest populationbased risk prediction modelling study to date, asthma has been found to be an independent risk factor for COVID-19 hospitalisations, but not death. ${ }^{16}$

The WHO declared COVID-19 a pandemic on 11 March $2020,{ }^{17}$ which triggered unprecedented nationally enforced social distancing measures by governments across the globe. These measures included widespread lockdowns of whole societies, including school closures and movement restrictions ${ }^{18}$ that have had numerous negative health, social and economic effects. ${ }^{19-22}$ These lockdowns have however had the beneficial effects of leading to improvements in air quality ${ }^{23-25}$ and a likely reduction in circulating respiratory viruses (especially in children and young people). It is also possible that the earlier concerns about increased risk of severe illness and death from COVID-19 may have led to improved self-management and shielding among patients with asthma. Consequently, we hypothesised that the UK's national lockdown, which began on 23 March 2020, lead to a reduction in asthma exacerbations in England. We tested our hypothesis by following a large cohort of patients with asthma using a national-level primary care database.

\section{METHODS}

\section{Data source and setting}

We used the Optimum Patient Care Database (OPCRD), a live and growing database of de-identified, longitudinal electronic medical records populated by a network of primary care practices from across the UK. OPCRD has been used to conduct epidemiological, pharmaceutical and clinical studies (https://opcrd.co.uk/). ${ }^{26} 27$ At the time of data access, OPCRD consisted of almost 10 million patients from 792 practices. We used data from the 670 practices in England (the remaining practices were from other nations of the UK). In addition to demographic information, the OPCRD dataset contains diagnoses, symptoms, treatment and prescription data encoded with Read codes, a comprehensive system of clinical concepts classification system that has been used in primary care practices across the UK for about three decades. ${ }^{28}$ For this study, we were provided with a secure access to the Microsoft SQL database through a secure remote access.

\section{Study design and population}

We identified a cohort of patients with clinician diagnosed and recorded asthma who were then followed over time to assess if and when they experienced asthma exacerbations. The cohort of patients with asthma was identified with a previously validated algorithm ${ }^{29}$ over the period 1 January 2010 to 31 December 2015. This algorithm comprised of 121 specific asthma codes that have previously been shown to identify patients with asthma from UK primary care records with high accuracy. ${ }^{29}$ The cohort of patients with asthma was then followed from 1 January 2016 to 16 August 2020; and the outcome measure, asthma exacerbations, was determined for every patient for each week. Using this cohort of patients, we designed an interrupted time-series study with control. The follow-up period was divided into a COVID-19 year (the 'intervention' period) and pre-COVID-19 years (the 'control' period). The single point intervention was the week corresponding to imposition of lockdown (23 March 2020); the control was the mean weekly exacerbation rate (mean of 4 years, 2016-2019 inclusive for January-August), and the intervention period was the weekly exacerbation rate from January to August 2020.

\section{Ascertainment of outcome}

Based on the American Thoracic Society/European Respiratory Society Task Force definition ${ }^{30}$ and previously validated in OPCRD,${ }^{31}$ an asthma exacerbation in a given assessment period was defined as the presence of either one of the following: an asthma-related accidental and emergency (A\&E) department attendance, an asthma-related hospital admission or an acute course of oral corticosteroids (OCS) with evidence of respiratory review within 2 weeks of OCS prescription. In addition to Read codes associated with asthma-associated hospital attendance or admission, we identified additional non-asthma-specific hospitalisation codes and considered a patient to have experienced a hospital-associated asthma exacerbation if a patient had any of the 121 specific asthma codes (previously used to identify patients with asthma ${ }^{29}$ ) on the same day when they had any hospitalisation code in their records. Online supplemental appendix 1 provides a list of the Read codes used to ascertain the outcome.

\section{Data analysis}

For every assessment period (defined as a week), we determined the total number of exacerbations, normalised this number with the total number of patients in the study in every period and then converted it into an exacerbation rate (total number of exacerbation episodes per patient-year). Once the exacerbation rate was derived for every week from January 2016 to August 2020, the data were split into a COVID-19 year and pre-COVID-19 years. In 2020, the pre-lockdown period corresponded to weeks 1-12 (January-March), and the post-lockdown period corresponded to the period starting from week 13 (week starting 23 March). Every year in the follow-up (2016-2019) was divided into two periods (weeks 1-12 and weeks 13-32) to allow a comparison of those years with the year 2020 when the national lockdown was imposed. Data were then analysed using an interrupted time-series design with control. First, ordinary least squares regression (OLS) analysis was applied with eight coefficients to be determined. The eight coefficients were: an intercept term and existing trend; existing level and trend difference; postintervention level and trend; and level change and trend change difference. The OLS model was then tested for the presence of 'autoregression' and 'regression' type relationships in the data (autocorrelation type relationships are expected in data with seasonality pattern) with autocorrelation and partial autocorrelation plots. This step also helped to determine the order of moving average and/or autoregression relationship in the data. Subsequently, a generalised least squares model was fitted to the data incorporating both the autoregression and moving average relationship in the data. The final fitted model was then used to extract the absolute and relative changes in the outcome of interest. In our study, the outcomes of interest were overall exacerbations, exacerbations that were resolved within primary care without any hospital visit, exacerbations that required a hospital visit, and overall exacerbation stratified by age groups, sex and region. Interrupted time-series analysis allows us to measure two potential changes that could occur because of the intervention: a change in level and a change in trend. Change in level corresponds to the sudden change in the exacerbation rate immediately after the intervention. A change in trend corresponds to difference in the trend (slow change of exacerbation rate over time) between pre-intervention and post-intervention periods.

All analyses were undertaken in $\mathrm{R}$ Studio (V.1.2.5033) using R (V.3.6.2). The tidyverse packages ${ }^{32}$ were used for data manipulation (dplyr), date manipulations (lubridate) and data 


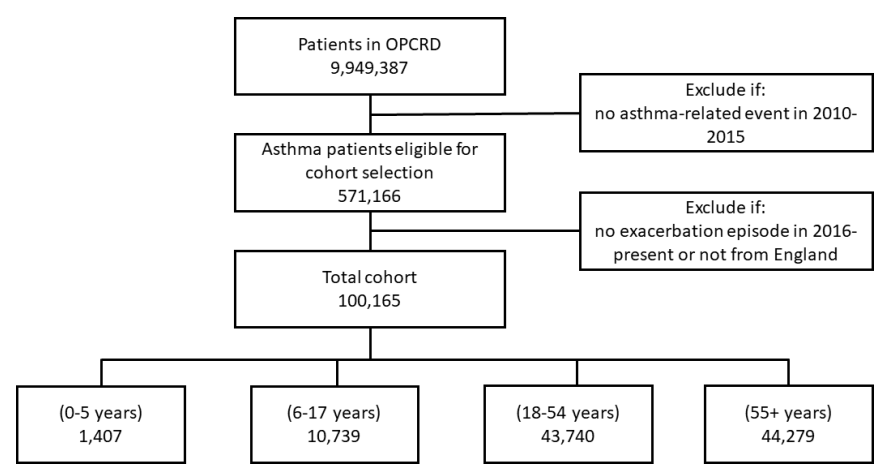

Figure 1 Overall flow diagram of patients in the study. OPCRD, Optimum Patient Care Database.

visualisations (ggplot2). The nlme package $^{33}$ was used for building the generalised least squares model. The final figures illustrating the results of the interrupted time series were plotted with Matlab (R2018a, Mathworks, USA). ${ }^{34}$

\section{Study reporting}

This study is reported following the recommendations of Reporting of studies Conducted using Observational Routinelycollected Data. ${ }^{35}$
RESULTS

\section{Baseline characteristics of the study population}

Out of the 571166 patients with asthma identified $15.7 \%$ of the total population in OPCRD), 100165 patients (17.5\% of those identified with asthma) had at least one exacerbation during the follow-up period (January 2016-August 2020). The total follow-up time in the study was 416639 patient-years (mean follow-up of 4.16 patient-years). Figure 1 provides a flow diagram of the patients in the study. There were more women $(60360 ; 60.1 \%)$ than men $(39673 ; 39.6 \%)$ with a small number with missing sex information $(132 ; 0.1 \%)$. Most patients in the study were those aged $18-54(43740 ; 43.7 \%)$ and $\geq 55$ years (44 279; 44.2\%). The number of young patients with asthma was relatively low: $0-5$ years $(1407 ; 1.4 \%), 5-18$ years $(10739$; $10.7 \%)$. A large proportion of the patients in the cohort were from East England (21 002; 21\%), South East (20 445; 20.4\%) and Yorkshire and the Humber (17 240; 17.2\%). The remaining patients were from East Midlands (4483; 4.5\%), London (3302; 3.3\%), North East (4985; 5.0\%), North West (12 456; 12.4\%), South West $(14378 ; 14.4 \%)$ and West Midlands (1874; 1.9\%).

\section{Exacerbation pattern during follow-up}

Figure 2 provides the mean exacerbation rate of the patient cohort for every week during the follow-up period (January 2016-August 2020) categorised by whether patients were

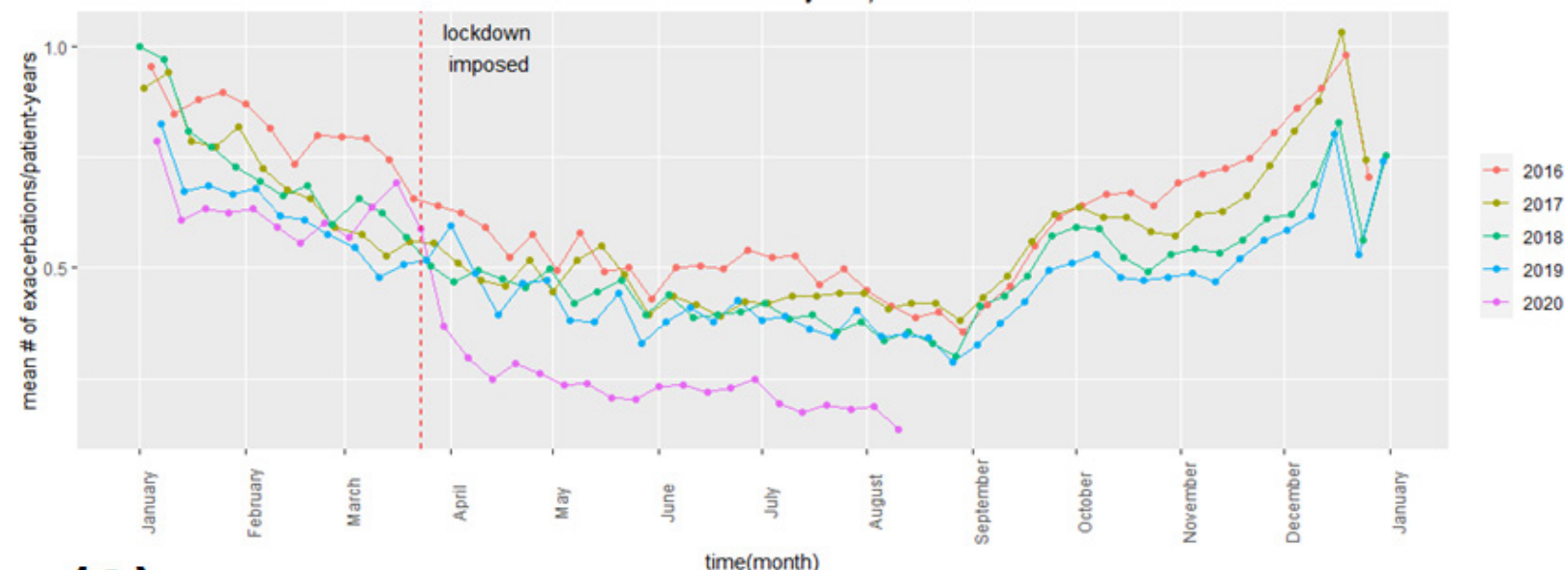

(A)

time(month)

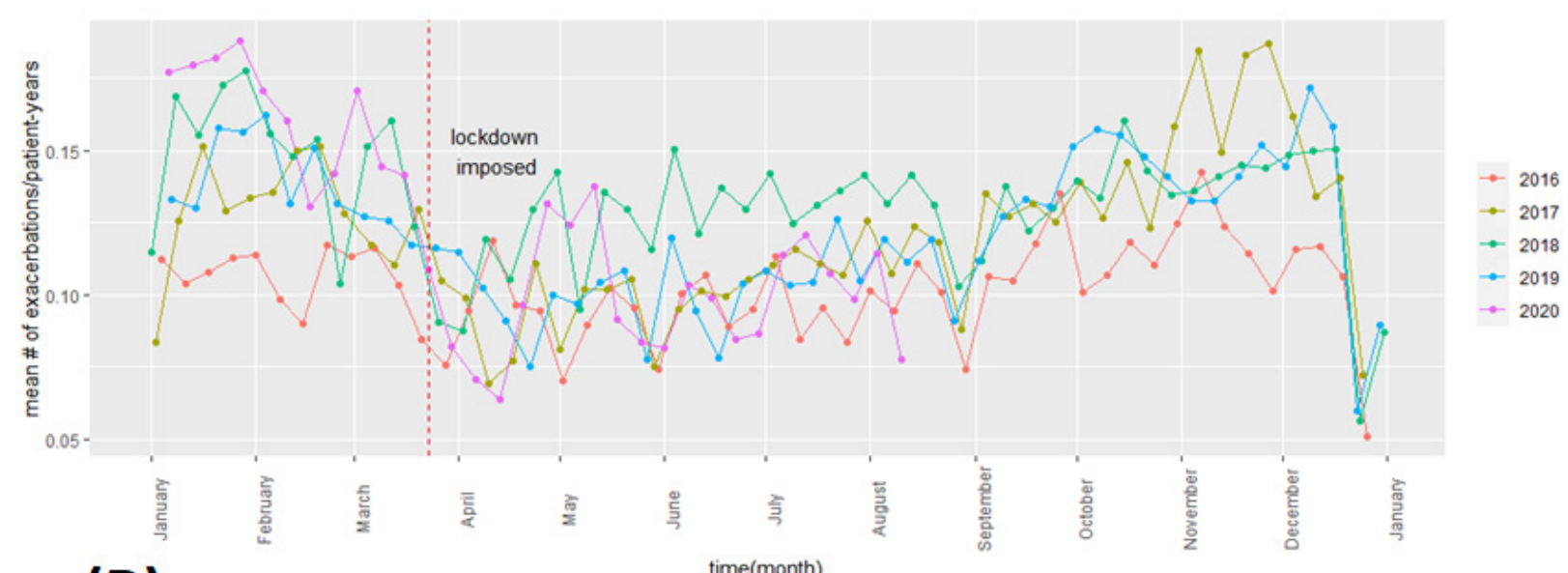

(B)

time(month)

Figure 2 Mean exacerbation rate for every week, from January 2016 to August 2020 across England stratified by exacerbation type: (A) primary care-based exacerbations only and (B) hospital-based exacerbations only. 
Table 1 Total number of patients and exacerbation rate over time (mean rates for each period given, weeks refer to ISO weeks)

\begin{tabular}{|c|c|c|c|c|}
\hline \multirow[b]{2}{*}{ Period } & \multirow[b]{2}{*}{ Number of patients in cohort } & \multicolumn{3}{|c|}{$\begin{array}{l}\text { Exacerbation rate (number of episodes/person-year) } \\
\text { mean }(95 \% \mathrm{Cl})\end{array}$} \\
\hline & & Resolved within primary care only & Required hospital visit & Overall \\
\hline 2016 (weeks 1-12) & 100165 & 0.82 (0.76 to 0.87$)$ & $0.11(0.10$ to 0.11$)$ & 0.92 (0.87 to 0.98$)$ \\
\hline 2016 (weeks 13-32) & 99813 & 0.52 (0.49 to 0.55 ) & $0.10(0.09$ to 0.10$)$ & $0.61(0.58$ to 0.64$)$ \\
\hline 2017 (weeks 1-12) & 98264 & $0.71(0.62$ to 0.80$)$ & $0.13(0.12$ to 0.14$)$ & $0.84(0.75$ to 0.93$)$ \\
\hline 2017 (weeks 13-32) & 97689 & 0.46 (0.43 to 0.48$)$ & 0.10 (0.09 to 0.11$)$ & 0.56 (0.53 to 0.58$)$ \\
\hline 2018 (weeks 1-12) & 96012 & 0.73 (0.64 to 0.82 ) & $0.15(0.13$ to 0.16$)$ & 0.88 (0.79 to 0.97$)$ \\
\hline 2018 (weeks 13-32) & 95410 & 0.43 (0.40 to 0.45$)$ & $0.13(0.12$ to 0.13$)$ & 0.55 (0.53 to 0.57$)$ \\
\hline 2019 (weeks 1-12) & 91439 & 0.62 (0.55 to 0.68$)$ & $0.14(0.13$ to 0.15$)$ & 0.75 (0.68 to 0.82$)$ \\
\hline 2019 (weeks 13-32) & 88976 & 0.41 (0.38 to 0.44 ) & 0.10 (0.10 to 0.11$)$ & 0.51 (0.48 to 0.54$)$ \\
\hline 2020 (weeks 1-12) & 77258 & 0.63 (0.59 to 0.67 ) & $0.16(0.14$ to 0.17$)$ & $0.78(0.74$ to 0.83$)$ \\
\hline 2020 (weeks 13-32) & 67995 & $0.23(0.20$ to 0.25$)$ & 0.10 (0.09 to 0.11$)$ & $0.33(0.30$ to 0.35$)$ \\
\hline
\end{tabular}

Total number of exacerbation episodes: 278 996; exacerbation episodes managed exclusively by a general practitioner: 229058 (82.1\%); exacerbation episodes requiring

hospital visit: 49938 (17.9\%).

managed by general practitioners (GPs) only (termed primary care based in the figure) or whether patients eventually attended hospital (termed hospital based in the figure, which included both A\&E attendance and hospital admission). The figure illustrates the seasonality pattern in both categories: the exacerbation rate gradually decreased from January onwards until summer, and it then gradually increased again from August/ September until December/January. The sharp decrease during the last week of December corresponded to restricted opening times of primary care practices. Online supplemental appendix 2 provides the mean exacerbation rate of all patients in the cohort during follow-up categorised by sex (online supplemental figure S1), age (online supplemental figure S2) and region (online supplemental figure S3).

During follow-up, there were a total of 278996 exacerbation episodes experienced by the 100165 patients. Of these, 229 $058(82.1 \%)$ were managed exclusively in primary care without requiring any hospital visit, and the remaining 49938 (17.9\%) required a hospital visit. Table 1 provides the mean exacerbation rate for the patients in the cohort in two distinct periods: weeks 1-12 and 13-32. There was a significant drop in the mean exacerbation rate for all years during the follow-up when comparing weeks 1-12 (corresponding to January-March) with weeks 13-32 (corresponding to April-August).

\section{Interrupted time-series analyses}

Table 2 provides results of the interrupted time-series analyses (the rates in this table express the number of exacerbations per person-year). The intercept (akin to a mean value when trend and change due to intervention is separately accounted for) in the table shows that: there were more exacerbations that did not require hospital attendance/admission and were resolved within primary care compared with those that required hospital attendance/admission (0.913 vs 0.134$)$; overall, women were slightly more likely to experience exacerbations than men $(1.090$ vs $0.976)$; and the exacerbation rate was highest in the $\geq 55$ age group (1.305) followed by 18-54 (0.919) and 0-5 (0.839) and lowest in 5-17 (0.618). Based on regions in England (as represented in OPCRD), West Midlands had the highest rate of exacerbation (1.383) followed by the North East (1.205), while London had the lowest rate of exacerbation (0.639).

Overall, there was a statistically significant change in level $(-0.196 ; p=0.008)$ of exacerbation rate. The change in trend was, however, not significant $(0.029 ; \mathrm{p}=0.054)$. When exacerbations were categorised into whether they were managed exclusively in primary care with no need of hospital attendance and/or admission, or whether they required hospital visit, we only found a statistically significant difference in level $(-0.244$; $\mathrm{p}=0.000)$ and trend $(-0.022 ; \mathrm{p}=0.000)$ in the former case. Figure 2 shows mean exacerbation rate for every week, from January 2016 to August 2020 across England stratified by exacerbation type: (a) primary care-based exacerbations only and (b) hospital-based exacerbations only.

Figure 3 illustrates the result of the interrupted time-series analyses for both of these categories. Table 2 provides results of the interrupted time-series analyses when the cohort was stratified by sex, age and region. Stratified by sex, there is a significant reduction in trend and level for both men and women. Stratified by age, there was a significant drop in all groups with the most notable decrease in the youngest age group (aged 5-10 years in 2020). When stratified by region, there was a significant drop in level across all regions except London and the North East.

\section{DISCUSSION}

We found a significant overall decrease in asthma exacerbations in England during the COVID-19 pandemic after the lockdown was imposed on 23 March 2020. The decrease was statistically significant in all age groups, in both men and women, and almost across all regions in England. The primary care dataset allowed us to assess whether a given exacerbation episode was resolved within primary care or whether it required a hospital visit. Throughout follow-up (January 2016-August 2020), most asthma exacerbations were resolved within primary care without requiring any hospital visit. We found a statistically significant drop in exacerbation episodes that did not require hospital visit, after the lockdown was imposed. There was no significant decrease in exacerbation episodes that required hospital visit. Overall, our findings indicate that there was a substantial decrease in asthma exacerbations that did not require hospital visit (likely mild cases), but insufficient evidence to suggest any impact on those (presumably more severe) cases of asthma exacerbations that required a hospital visit.

To our knowledge, this is the first national-level study that assessed the impact of the COVID-19 pandemic on attendance to primary or secondary care for asthma exacerbations. 
Table 2 Intercept, residual SE, and change in level and trend after lockdown was imposed with corresponding p values (compared with average of last 4 years, the control group)

\begin{tabular}{|c|c|c|c|c|}
\hline Cohort & Intercept & Change in level after intervention & Change in trend after intervention & Residual SE \\
\hline All patients, $\mathrm{n}=100165$ & $0.833^{*}$ & $-0.196(0.0077)$ & $0.029(0.0536)$ & 0.082 \\
\hline \multicolumn{5}{|l|}{ Stratification by healthcare setting } \\
\hline Resolved within primary care & $0.913^{*}$ & $-0.244^{*}$ & $-0.022(0.0001)$ & 0.032 \\
\hline Hospital & $0.134^{*}$ & $-0.005(0.7894)$ & $0.004(0.1149)$ & 0.014 \\
\hline \multicolumn{5}{|l|}{ Stratification of population cohort by sex $t$} \\
\hline Men, $n=39673$ & $0.976^{*}$ & $-0.210^{*}$ & $-0.009(0.0068)$ & 0.035 \\
\hline Women, $\mathrm{n}=60360$ & $1.090^{*}$ & $-0.277^{*}$ & $-0.024(0.0015)$ & 0.042 \\
\hline \multicolumn{5}{|c|}{ Stratification of population cohort by age (years) } \\
\hline $0-5, n=1407$ & $0.839^{*}$ & $-0.367(0.0013)$ & $-0.009(0.5175)$ & 0.127 \\
\hline $5-17, n=10739$ & $0.618^{*}$ & $-0.159(0.0328)$ & $-0.005(0.6351)$ & 0.061 \\
\hline $18-54, n=43740$ & $0.919^{*}$ & $-0.238(0.0010)$ & $-0.037(0.0004)$ & 0.059 \\
\hline$\geq 55, n=44279$ & $1.305^{*}$ & $-0.241^{*}$ & $0.002(0.7942)$ & 0.041 \\
\hline \multicolumn{5}{|l|}{ Stratification of population cohort by region } \\
\hline East England, $\mathrm{n}=21002$ & $1.067^{*}$ & $-0.283^{*}$ & $-0.014(0.0307)$ & 0.048 \\
\hline East Midlands, $\mathrm{n}=4483$ & $0.787^{*}$ & $-0.371^{*}$ & $-0.037(0.0003)$ & 0.083 \\
\hline London, $\mathrm{n}=3302$ & $0.639^{*}$ & $-0.261(0.0702)$ & $-0.004(0.8451)$ & 0.133 \\
\hline North East, $n=4985$ & $1.205^{*}$ & $-0.068(0.6176)$ & $-0.025(0.1845)$ & 0.108 \\
\hline North West, $n=12456$ & $1.186^{*}$ & $-0.258(0.0147)$ & $-0.060(0.0001)$ & 0.089 \\
\hline South East, $n=20445$ & $1.030^{*}$ & $-0.261^{*}$ & $0.000(0.9582)$ & 0.051 \\
\hline South West, n=14 378 & $1.049^{*}$ & $-0.275^{*}$ & $-0.010(0.0028)$ & 0.050 \\
\hline West Midlands, $\mathrm{n}=1874$ & $1.383^{*}$ & $-0.590(0.0031)$ & $-0.146^{*}$ & 0.191 \\
\hline Yorkshire and the Humber, $\mathrm{n}=17240$ & $0.989^{*}$ & $-0.132(0.0235)$ & $-0.011(0.1555)$ & 0.048 \\
\hline
\end{tabular}

${ }^{*} \mathrm{P}<0.0001$.

tA total of 132 patients did not have sex information in the database.

The key strengths of this study are a long follow-up (January 2016-August 2020), and the use of validated algorithms to identify patients with asthma and asthma exacerbations in a national primary care database from multiple centres covering a wide geographical area. This study used all English data in the OPCRD that were collected as part of routine clinical care pathway thereby minimising both selection and information bias often associated with observational studies. ${ }^{36}$

There are some limitations to our study. We only had access to primary care records and while primary care will be the first point of contact for any patient with asthma, it is likely that some patients might have attended A\&E department in hospital without referral. Our study may miss any such episodes, particularly if there is no subsequent primary care follow-up by the patient or if there is no communication between primary and secondary care. While it is not possible to ascertain the number of such episodes due to absence of any linked data (to link primary care records with A\&E), we believe that such occurrences will be rare since patients with chronic condition (such as asthma) are likely to make contact with primary care for follow-up and/or medication. In addition, a discharge letter is typically sent to primary care following any hospital discharge which then gets added to a patient's primary care record. Second, while interrupted time-series analyses quantitatively show the extent of difference observed after an intervention, it cannot ascertain any causal relationships. Lastly, we have assumed that the approach of GPs in using the Read codes to manage patients with asthma has not changed during the pandemic.

It is important to highlight that our analysis included all patients with asthma who had at least one exacerbation during the follow-up period. While we could include all patients in the analysis, it will not change the results of the interrupted time-series analysis. This is because these excluded patients had zero exacerbation episodes and therefore the relative change in exacerbation rate from pre-COVID-19 years to COVID-19 years will not change (only the absolute value of the exacerbation rate, and hence the intercept value in the interrupted time-series analysis will change across the whole time series).

Since viral respiratory infections are the main trigger of asthma exacerbations, ${ }^{10}$ there were concerns at the start of the pandemic that COVID-19 may have led to increase in asthma exacerbations and that COVID-19 outcomes would be poorer in those with asthma. Several studies, to date, have investigated COVID-19 outcomes in patients with asthma. ${ }^{13} 161819212533$ However, to date, the impact of the pandemic on asthma exacerbations is unclear. A recent study by Public Health England investigating causes of excess public deaths found a reduction in respiratory-related deaths (acute respiratory infections, chronic lower respiratory diseases and other respiratory diseases) during the pandemic when compared with deaths from the previous 5 years in England. ${ }^{37}$ Another recent study that aimed to derive and validate a risk prediction algorithm to estimate COVID-19 


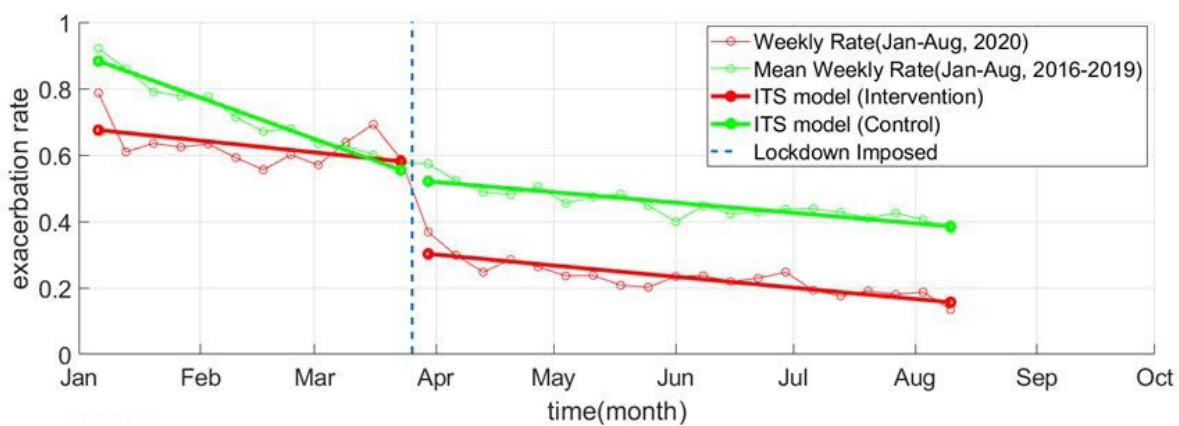

(A)

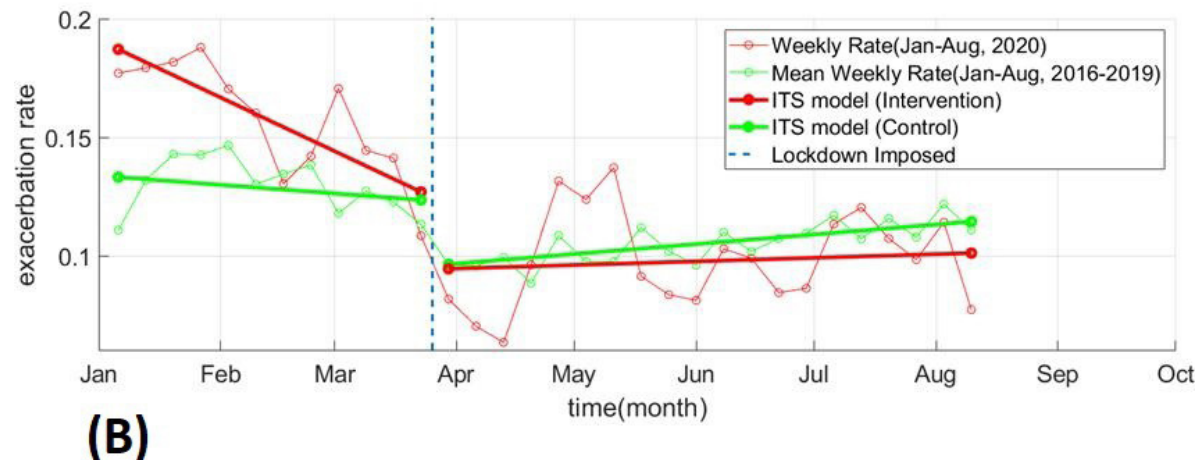

Figure 3 Interrupted time-series (ITS) model fitted to yearly exacerbation rate for every week from January to August for control group (mean rate from 2016 to 2019) and the intervention group (2020): (A) exacerbations related to primary care consultations only and (B) hospital-based exacerbations only.

outcomes found that asthma does not increase risk of dying in either men or women, and only marginally increases the risk hospitalisation. ${ }^{16}$ These recent results support a reassuring viewpoint that patients with asthma are, perhaps, not as high risk as initially feared, and these studies align with our results that suggest an overall decrease in asthma exacerbations.

One possible explanation for a significant decrease only in exacerbation episodes that did not require hospital visit could be due to a change in careseeking behaviour. Some patients may have preferred exacerbating at home rather than reporting to primary care for fear of COVID-19 and a subset of those patients whose exacerbation did not resolve at home ended up visiting hospital for their asthma. It is also possible that some patients may have preferred self-referring themselves to A\&E, as opposed to contacting primary care, so that they could be seen in person (in response to the pandemic, there was a shift from in-person to remote consultations in primary care across the $\left.\mathrm{UK}^{38}\right)$. However, by and large, there was an overall decrease in asthma exacerbations and while our study cannot definitively prove the reasons for this decrease, we believe that a combination of factors led to a reduction in asthma exacerbations. These factors include changing behaviour due to lockdown measures leading to reduction in air pollution, reduced circulation of respiratory viruses, improved self-management driven by patient concerns during the pandemic ${ }^{39}$ and shielding by a subset of patients (government-introduced UK-wide scheme where high-risk individuals are contacted and advised to follow stricter restrictions than the rest of the population ${ }^{40}$ ). We cannot, however, ascertain the role, if any, and extent of these factors in the current study. There is now a need to undertake further research (surveys of patient's attitudes, and qualitative interviews with patients and primary care health professionals) to understand the social and disease-related mechanisms that can help explain our findings.

\section{CONCLUSION}

Our study, the largest cohort study assessing the impact of COVID-19 on asthma to date, showed that there has been a significant reduction in asthma exacerbations (as recorded in primary care) during the pandemic and this reduction was observed in all age groups, both men and women and across most regions in England. This reduction in exacerbation rate was mainly seen in relation to exacerbations resolved within primary care without a need for a hospital visit. There is a need for further work to investigate the factors responsible for this decrease.

\section{Twitter Aziz Sheikh @DrAzizSheikh}

Acknowledgements We acknowledge the support of OPC (https://opcrd.co.uk) who have provided us with access to their database through agreement with Asthma UK Centre for Applied Research (www.aukcar.ac.uk). This project is based wholly on Data from the Optimum Patient Care Research Database obtained under licence from Optimum Patient Care Limited and its execution is approved by recognised experts affiliated to the Respiratory Effectiveness Group. However, the interpretation and conclusions contained in this report are those of the authors alone. Access to OPCRD was funded by Optimum Patient Care (OPC[SH6]).

Contributors AS conceived the initial idea. SAS with JKQ, BIN and AS designed the study protocol. SAS accessed and analysed the data and wrote the initial draft of the manuscript. All coauthors contributed to and approved the final version of the manuscript.

Funding This work was supported by BREATHE-The Health Data Research Hub for Respiratory Health (MC_PC_19004), the National Institute for Health Research (NIHR) Imperial Biomedical Research Centre (BRC) and the University of Edinburgh's Chancellor's Fellowship Scheme. BREATHE is funded through the UK Research and Innovation Industrial Strategy Challenge Fund and delivered through Health Data Research UK. 
Disclaimer The views expressed are those of the authors and not necessarily those of the NIHR or the Department of Health and Social Care.

Competing interests None declared.

Patient consent for publication Not required.

Ethics approval The study was approved by the Anonymized Data Ethics and Protocol Transparency committee (reference number: ADEPT1020), which grants project-specific approvals for use of OPCRD data. Optimum Patient Care has an existing NHS Health Research Authority ethics approval for use of OPCRD for research (REC Ref: 15/EM/150). All researchers involved in data analysis completed required information governance courses before working on the data.

Provenance and peer review Not commissioned; externally peer reviewed.

Data availability statement Data may be obtained from a third party and are not publicly available. The dataset for this work can be accessed from OPCRD (https://opcrd.co.uk/) subject to protocol approval from an independent scientific advisory committee, ADEPT (Anonymized Data Ethics and Protocol Transparency committee). The code used in the analysis in our current work will be made available at https://github.com/syedahmar/Covid19-ImpactAsthmaExacerbations under Creative Commons License.

Open access This is an open access article distributed in accordance with the Creative Commons Attribution 4.0 Unported (CC BY 4.0) license, which permits others to copy, redistribute, remix, transform and build upon this work for any purpose, provided the original work is properly cited, a link to the licence is given, and indication of whether changes were made. See: https://creativecommons.org/ licenses/by/4.0/.

ORCID iDs

Jennifer K Quint http://orcid.org/0000-0003-0149-4869

Bright I Nwaru http://orcid.org/0000-0002-2876-6089

\section{REFERENCES}

1 Wang D, Hu B, Hu C, et al. Clinical characteristics of 138 hospitalized patients with 2019 novel coronavirus-infected pneumonia in Wuhan, China. JAMA 2020;323:1061-9.

2 Jordan RE, Adab P, Cheng KK. Covid-19: risk factors for severe disease and death. BMJ 2020;368:m1198.

3 Garg S, Kim L, Whitaker M, et al. Hospitalization Rates and Characteristics of Patients Hospitalized with Laboratory-Confirmed Coronavirus Disease 2019 - COVID-NET, 14 States, March 1-30, 2020. MMWR Morb Mortal Wkly Rep 2020;69:458-64.

4 Richardson S, Hirsch JS, Narasimhan M, et al. Presenting characteristics, comorbidities, and outcomes among 5700 patients hospitalized with COVID-19 in the new York City area. JAMA 2020;323:2052.

5 Docherty AB, Harrison EM, Green CA, et al. Features of 20133 UK patients in hospital with covid-19 using the ISARIC WHO Clinical Characterisation Protocol: prospective observational cohort study. BMJ 2020;369:m1985.

6 Williamson EJ, Walker AJ, Bhaskaran K, et al. Factors associated with COVID-19related death using OpenSAFELY. Nature 2020;584:430-6.

7 Reilev M, Kristensen KB, Pottegård A, et al. Characteristics and predictors of hospitalization and death in the first 11122 cases with a positive RT-PCR test for SARS-CoV-2 in Denmark: a nationwide cohort. Int J Epidemiol 2020;49:1468-81.

8 Ivanova Jl, Bergman R, Birnbaum HG, et al. Effect of asthma exacerbations on health care costs among asthmatic patients with moderate and severe persistent asthma. J Allergy Clin Immunol 2012;129:1229-35.

9 Mukherjee M, Stoddart A, Gupta RP, et al. The epidemiology, healthcare and societal burden and costs of asthma in the UK and its member nations: analyses of standalone and linked national databases. BMC Med 2016;14:113.

10 Johnston NW, Sears MR. Asthma exacerbations . 1: epidemiology. Thorax 2006;61:722-8.

11 C. for D. C. and Prevention. Coronavirus disease 2019 (COVID-19), 2020. Available: https://www.cdc.gov/coronavirus/2019-ncov/need-extra-precautions/asthma.html [Accessed 23 Oct 2020].

12 Li X, Xu S, Yu M, et al. Risk factors for severity and mortality in adult COVID-19 inpatients in Wuhan. J Allergy Clin Immunol 2020;146:110-8.

13 Beurnier A, Jutant E-M, Jevnikar M, et al. Characteristics and outcomes of asthmatic patients with COVID-19 pneumonia who require hospitalisation. Eur Respir J 2020;56:2001875
14 Goyal P, Choi JJ, Pinheiro LC, et al. Clinical characteristics of Covid-19 in New York City. N Engl J Med 2020;382:2372-4.

15 Chhiba KD, Patel GB, Vu THT, et al. Prevalence and characterization of asthma in hospitalized and nonhospitalized patients with COVID-19. J Allergy Clin Immunol 2020;146:307-14.

16 Clift AK, Coupland CAC, Keogh RH, et al. Living risk prediction algorithm (QCOVID) for risk of hospital admission and mortality from coronavirus 19 in adults: national derivation and validation cohort study. BMJ 2020;371:m3731.

17 Cucinotta D, Vanelli M. "WHO declares COVID-19 a pandemic. "Acta bio-medica Atenei Parm 2020;91:157-60.

18 Hale T, Webster S, Petherick A. Oxford covid-19 government response tracker. Blavatnik Sch. Gov 2020;25.

19 Rossi Ret al. COVID-19 pandemic and lockdown measures impact on mental health among the general population in Italy. An $\mathrm{N}=18147$ web-based survey. medRxiv 2020.

20 Bhuiyan AKMI, Sakib N, Pakpour AH, et al. COVID-19-Related suicides in Bangladesh due to Lockdown and economic factors: case study evidence from media reports. Int $J$ Ment Health Addict 2020;39:1-6.

21 Payne S. Coronavirus: the hidden health costs of the UK lockdown. London: Financial Times, 2020.

22 Triggle N. Coronavirus: what is the hidden health cost? London: BBC, 2020.

23 Bao R, Zhang A. Does lockdown reduce air pollution? Evidence from 44 cities in northern China. Sci Total Environ 2020;731:139052.

24 Kerimray A, Baimatova N, Ibragimova OP, et al. Assessing air quality changes in large cities during COVID-19 lockdowns: the impacts of traffic-free urban conditions in Almaty, Kazakhstan. Sci Total Environ 2020;730:139179.

25 Muhammad S, Long X, Salman M. COVID-19 pandemic and environmental pollution: a blessing in disguise? Sci Total Environ 2020;728:138820.

26 Jones RCM, Price D, Ryan D, et al. Opportunities to diagnose chronic obstructive pulmonary disease in routine care in the UK: a retrospective study of a clinical cohort. Lancet Respir Med 2014;2:267-76.

27 Price DB, Rigazio A, Campbell JD, et al. Blood eosinophil count and prospective annua asthma disease burden: a UK cohort study. Lancet Respir Med 2015;3:849-58.

28 Chisholm J. The read clinical classification. BMJ 1990;300:1092.

29 Nissen F, Morales DR, Mullerova H, et al. Validation of asthma recording in the clinical practice research Datalink (CPRD). BMJ Open 2017;7:e017474.

30 Reddel HK, Taylor DR, Bateman ED, et al. An official American thoracic Society/ European respiratory Society statement: asthma control and exacerbations: standardizing endpoints for clinical asthma trials and clinical practice. Am J Respir Crit Care Med 2009;180:59-99.

31 Colice G, Chisholm A, Dima AL, et al. Performance of database-derived severe exacerbations and asthma control measures in asthma: responsiveness and predictive utility in a UK primary care database with linked questionnaire data. Pragmatic Obs. Res 2018;9:29-42.

32 Wickham H, Averick M, Bryan J, et al. Welcome to the Tidyverse. J Open Source Softw 2019:4:1686.

33 Pinheiro Jet al. "Package 'nlme,'" Linear nonlinear Mix. Eff. Model. version 32017.

34 MATLAB V. 9.4. 0 (R2018a. Natick, MA, USA: MathWorks Inc, 2018.

35 Benchimol El, Smeeth L, Guttmann A, et al. The reporting of studies conducted using observational Routinely-collected health data (record) statement. PLoS Med 2015; 12:e1001885.

36 Hammer GP, du Prel J-B, Blettner M. Avoiding bias in observational studies: part 8 in a series of articles on evaluation of scientific publications. Dtsch Arztebl Int 2009:106:664.

37 Public Health England. Excess mortality in England, week ending 18 September 2020, 2020. Available: https://fingertips. phe.org.uk/static-reports/mortalitysurveillance/excess-mortality-in-england-week-ending-18-Sep-2020.html [Accessed 08 Oct 2020].

38 Khan N, Jones D, Grice A, et al. A brave new world: the new normal for general practice after the COVID-19 pandemic. BJGP Open 2020;4:bjgpopen20X101103. doi:10.3399/bjgpopen20X101103

39 Kaye L, Theye B, Smeenk I, et al. Changes in medication adherence among patients with asthma and COPD during the COVID-19 pandemic. J Allergy Clin Immunol Pract 2020;8:2384-5.

40 UK Government,. COVID-19: guidance on shielding and protecting people defined on medical grounds as extremely vulnerable, 2020. Available: https://www.gov.uk/ government/publications/guidance-on-shielding-and-protecting-extremely-vulnerablepersons-from-covid-19 